\title{
Occupational asthma, rhinitis and urticaria due to piperacillin sodium in a pharmaceutical worker
}

\author{
G. Moscato, E. Galdi, J. Scibilia, A. Dellabianca, P. Omodeo, \\ G. Vittadini, G.P. Biscaldi
}

Occupational asthma, rhinitis and urticaria due to piperacillin sodium in a pharmaceutical worker. G. Moscato, E. Galdi, J. Scibilia, A. Dellabianca, P. Omodeo, G. Vittadini, G.P. Biscaldi. OERS Journals Ltd 1995.

ABSTRACT: A 28 year old man with no history of atopy was referred to our hospital for possible work-related asthma. He had been employed in the production section of a pharmaceutical company for $2 \mathrm{yrs}$, and in the last 2 months he had complained of dyspnoea, wheezing, chest tightness, symptoms of rhinitis and a cutaneous rash when exposed to powdered antibiotics. Symptoms disappeared after being transferred to the packaging section.

When the subject was admitted to our department he was asymptomatic. Basal lung function tests were in the normal range. Bronchial challenges with methacholine and with ultrasonically-nebulized distilled water were negative. Skin-prick test with piperacillin sodium gave a strong positive response at a very low concentration. Specific inhalation challenge with piperacillin sodium resulted in an immediate asthmatic reaction, and also reproduced rhinitis symptoms and the cutaneous rash. A control challenge with lactose, and the specific challenge test with cefuroxime sodium (another antibiotic to which the patient was exposed at the workplace) were negative.

We conclude that piperacillin sodium is an agent that can cause occupational asthma.

Eur Respir J., 1995, 8, 467-679.
Specialization School of Allergology and Clinical Immunology, University of Pavia; Clinica del Lavoro Foundation, IRCCS, Medical Center of Pavia, Respiratory and Occupational Allergy Unit, Italy.

Correspondence: G. Moscato Clinica del Lavoro Foundation IRCCS Via Boezio 26 27100 Pavia Italy

Keywords: Antibiotics bronchial challenge low molecular weight compounds occupational asthma piperacillin sodium

Received: July 221994

Accepted after revision November 201994

\section{Case report}

A 28 year old man was referred to our department because of suspected occupational asthma. The subject had worked as a driver from the age of 23 to $25 \mathrm{yrs}$. In the last 30 months he had worked in a pharmaceutical company. He was first assigned to work in the production department, where different drugs, including cefuroxime sodium and piperacillin sodium (a semi-synthetic broad-spectrum penicillin) (fig. 1) were produced. His job consisted of pouring the drug powders from a can into the feed chute of the machine, which later poured small quantities of the powder in vials. He also checked that the machine operated properly. The production process was enclosed and the machine was equipped with local exhaust fans on the feed chute for lowering the dispersed powder concentration. During his work, the subject wore protective clothing which did not protect the mouth and nose. The mouth and nose were covered by a mask (without particle filter), the eyes were protected by goggles, and the hands by gloves.

Twenty two months after starting his job, the patient noticed the appearance of a cutaneous rash, sneezing, rhinorrhoea, nasal stuffiness and itching whilst present at the workplace. On one occasion, outside the production department, whilst handling an apparently empty container, which had been previously been filled with piperacillin sodium powder, he experienced an acute attack of dyspnoea, wheezing and thoracic tightness. A similar episode was experienced whilst passing through the room where the vials were washed by other employees. The patient experienced a few other less severe episodes of wheezing and chest tightness when passing in the vicinity of the room where the vials were cleaned. On all occasions, symptoms disappeared about $1 \mathrm{~h}$ after the onset if the patient left the workplace. The symptoms did not appear during weekends away from work or on holidays. He had never complained of these symptoms before being employed in this job. Because of these symptoms, the patient was transferred to the packaging department 2 months later. After having changed duties the symptoms disappeared.

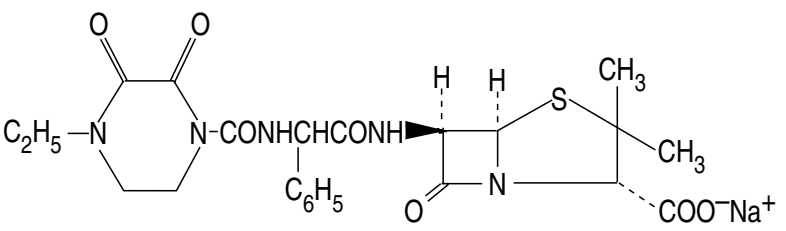

Fig. 1. - Chemical structure of piperacillin sodium 
The patient came to our observation 6 months after having been transferred to the packaging section. When he was admitted to our clinic, he did not have symptoms. He had no previous personal or family history of allergic disease. No abnormality was found in the physical examination. Basal lung function tests showed forced expiratory volume in one second $\left(\mathrm{FEV}_{1}\right) 106 \%$ predicted and vital capacity (VC) $93 \%$ predicted. Skin-prick tests and the assessment of serum specific immunoglobulin E (IgE) levels for the more common pneumoallergens were negative. Total IgE was in the normal range. Determination of serum specific IgE by radio allergosorbent test (RAST) for benzylpenicillin, phenoxymethylpenicillin, acetylsalicylic acid, aminopyrine, and trimethoprim sulphamethoxazole was also negative. The provocative dose of methacholine causing a $20 \%$ fall in $\mathrm{FEV}_{1}\left(\mathrm{PD}_{20 \mathrm{FEV}}\right)$ [1] was greater than $3.4 \mathrm{mg}$, indicating a normal bronchial responsiveness, and ultrasonically-nebulized distilled water challenge [1] was negative. The skin-prick test with piperacillin sodium at a concentration of $0.5 \mu \mathrm{g} \cdot \mathrm{ml}^{-1}$ was strongly positive (wheal size $5 \mathrm{~mm})$ [2].

The specific bronchial challenge was preceded by a control challenge (Day 1), in which the patient was requested to pour $50 \mathrm{~g}$ of lactose from one tray into another in a closed, controlled environment [3] for 20 min. Spirometry was performed before and at 5, 15 and $30 \mathrm{~min}$, and then hourly for $7 \mathrm{~h}$ after exposure. At the same time-points peak expiratory flow (PEF) was registered [4]. After $7 \mathrm{~h}, \mathrm{PEF}$ registration was continued by the patient himself throughout the evening (every hour), with the recommendation of measuring it at any time of symptom occurrence. Baseline $\mathrm{FEV}_{1}$ was 4.42 $l(105 \%$ pred $)$ and forced vital capacity was $4.86 l(97 \%$ pred). On Day 1, no significant variation of the pulmonary function (spirometry and/or PEF) was observed (fig. 2). On Day 2, baseline pulmonary function was unchanged, and the patient was exposed to $1 \mathrm{~g}$ of cefuroxime sodium in powder form mixed with $49 \mathrm{~g}$ of lactose,

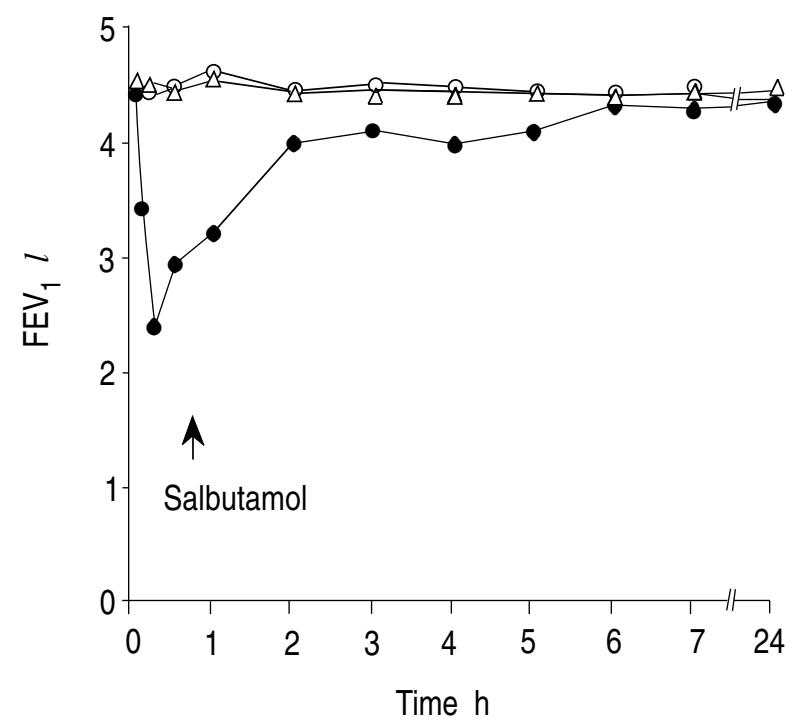

Fig. 2. - Bronchial challenge with lactose ( $\bigcirc)$, cefuroxime sodium ( $\boldsymbol{\Delta}$ ) and piperacillin sodium ( $)$ ). with the same method of pouring from one tray to another for $20 \mathrm{~min}$ in the same closed, controlled environment. No significant variation of the pulmonary function was observed at the same time-points of the control challenge (fig. 2). On Day 3, the patient underwent specific bronchial challenge with $1 \mathrm{~g}$ of piperacillin sodium in powder mixed with $49 \mathrm{~g}$ of lactose, as described above for cefuroxime. Five minutes after starting the exposure, the patient experienced symptoms of rhinitis, bronchospasm and cutaneous rash. The challenge was interrupted, and $15 \mathrm{~min}$ after the end of exposure a $45 \%$ fall in $\mathrm{FEV}_{1}$ was observed (fig. 2). No late reaction was observed in the laboratory up to $7 \mathrm{~h}$ or during the subsequent evening and night.

Occupational asthma, rhinitis, and urticaria caused by piperacillin sodium were confirmed, and the patient was discharged with the recommendation to avoid any further exposure to piperacillin sodium. At the 1, 3 and 6 month follow-up visits, the patient was completely free of symptoms and pulmonary function was within the normal range. He reported that he had no longer been exposed to the sensitizing agent.

\section{Discussion}

The patient history, the response to the skin-prick test and to the specific bronchial challenge with piperacillin sodium fulfilled the criteria for the diagnosis of occupational asthma, rhinitis, and urticaria due to piperacillin sodium. The causal relationship between symptoms and occupational exposure was also confirmed by the complete disappearance of symptoms after removal from exposure.

Among low molecular weight chemicals, antibiotics are well-known as agents able to induce occupational asthma in exposed workers [5-12]. In particular, the causal role of penicillin and its derivatives has been proved by means of specific bronchial challenges [5, 6]. To our knowledge, this is the first case described of occupational asthma caused by piperacillin sodium.

The features of the relationship between workplace exposure and symptoms in this patient are interesting. During his job inside the production department, the patient developed only nasal and cutaneous symptoms, but had no asthma attacks. The latter appeared outside the production department, when the patient was occasionally exposed to small quantities of piperacillin sodium powder. This might be explained by the fact that, as in most pharmaceutical industries, the production process was enclosed and the worker wore protective clothing and a mask. It is likely that these measures of exposure-control and protective devices employed in order to avoid massive contact with the offending agent were enough to prevent the onset of severe respiratory symptoms, but allowed allergic sensitization. As a consequence of sensitization, the exposure to even small quantities of piperacillin sodium outside the production department, where the subject was unprotected by a mask, resulted in acute asthma attacks.

As reported in several cases of occupational asthma [13], at the time of diagnosis bronchial responsiveness 
to methacholine and ultrasonic "fog" was normal. This may be accounted for by the fact that when the patient came to us for observation and treatment he had already been removed from direct exposure to the offending agent at work. The bronchial challenge with piperacillin sodium reproduced both the respiratory and the cutaneous symptoms experienced by the patient at the workplace. Symptom time-course was also similar: symptoms appeared immediately after handling the powder, and disappeared one hour after the end of exposure. The specificity of the response to piperacillin sodium is shown by the absence of response to lactose and to other antibiotics to which the patient was exposed at the workplace (cefuroxime sodium).

In contrast with other penicillin derivatives $[6,7]$, the strong positive cutaneous response to skin-prick test with a very diluted solution of piperacillin sodium provides evidence for an IgE-mediated mechanism. Although a RAST for piperacillin sodium was not available, several clinical features, such as the presence of a latency period between the beginning of occupational exposure and the onset of symptoms, the symptom time-course at the workplace, the type of asthmatic response to the specific bronchial challenge (immediate), and the simultaneous presence of a nasal and cutaneous response, support the presence of a type I mechanism.

In conclusion, we have demonstrated a case of occupational asthma, rhinitis and urticaria due to inhalation of piperacillin sodium, a semi-synthetic penicillin. This antibiotic should be listed as one of the agents that causes occupational asthma.

\section{References}

1. Moscato G, Dellabianca A, Falagiani P, Mistrello G, Rossi GL, Rampulla C. Inhaled furosemide prevents both the bronchoconstriction and the increase in neutrophil chemotactic activity induced by ultrasonic "fog" of distilled water in asthmatics. Am Rev Respir Dis 1991; 143: 561-566.

2. Dreborg S, ed. Skin tests used in type I allergy testing. Position paper. Allergy 1989; 44: (Suppl. 10): 38-46.

3. Moscato G, Pozzoli L. Presentazione di una cabina per test di broncostimolazione: comunicazione preliminare riguardante gli aspetti tecnici. G It Med Lav 1980; 2: 167-170.

4. Moscato G, Dellabianca A, Paggiaro PL, Bertoletti R, Corsico A, Perfetti L. Peak expiratory flow monitoring and airway response to specific bronchial provocation tests in asthmatics. Monaldi Arch Chest Dis 1993; 48: $23-28$.

5. Davies RJ, Hendrick DJ, Pepys J. Asthma due to inhaled chemical agents: ampicillin, benzylpenicillin, 6-amino penicillanic acid and related substances. Clin Allergy 1974; 4: 227-247.

6. Lagier F, Cartier A, Dolovich J, Malo J-L. Occupational asthma in a pharmaceutical worker exposed to penicillamine. Thorax 1989; 44: 157-158.

7. Coutts II, Dally MB, Newman-Taylor AJ, Pickering CAC, Horsfield N. Asthma in workers manufacturing cephalosporins. Br Med J 1981; 283: 950.

8. Briatico-Vangosa G, Beretta F, Bianchi S, et al. Bronchial asthma due to 7-aminocephalosporanic acid (7-ACA) in workers employed in cephalosporin production. Med Lav 1981; 72: 488-493.

9. Kammermeyer JK, Matthews KP. Hypersensitivity to phenylglycine acid chloride. J Allergy Clin Immunol 1973; 52: 73-84.

10. Davies RJ, Pepys J. Asthma due to inhaled chemical agents: the macrolide antibiotic spiramycin. Clin Allergy 1975; 1: 99-107.

11. Malo J-L, Cartier A. Occupational asthma in workers of a pharmaceutical company processing spiramycin. Thorax 1988; 43: 371-377.

12. Moscato G, Naldi L, Candura F. Bronchial asthma due to spiramycin and adipic acid. Clin Allergy 1984; 14 : 355-361.

13. Subcommittee on Occupational Allergy of the European Academy of Allergology and Clinical Immunology. Guidelines for the diagnosis of occupational asthma. Clin Exp Allergy 1992; 19: 397-401. 